\title{
Size-Controlled Synthesis of Near-Monodisperse Gold Nanoparticles in the 1-4 nm Range using Polymeric Stabilizers
}

Irshad Hussain, ${ }^{1,2}$ Susan Graham, ${ }^{1}$ Zhenxin Wang, ${ }^{1}$ Bien Tan, ${ }^{1}$ David C. Sherrington, ${ }^{3}$ Steven P. Rannard, ${ }^{1,4}$ Andrew I. Cooper, ${ }^{* 1}$ and Mathias Brust ${ }^{*, 1}$

\footnotetext{
${ }^{1}$ The University of Liverpool, Centre for Nanoscale Science, Department of Chemistry, Crown Street, Liverpool, L69 7ZD, United Kingdom. ${ }^{2}$ National Institute for Biotechnology and Genetic Engineering (NIBGE), Jhang Road Faisalabad, Pakistan. ${ }^{3}$ The Department of Pure and Applied Chemistry, Thomas Graham Building, University of Strathclyde, 295 Cathedral Street, Glasgow G1 1XL, Scotland.

${ }^{4}$ Unilever Research, Port Sunlight Laboratory, Quarry Road East, Bebington, Wirral, L63 3JW, UK.
}

\section{SUPPORTING INFORMATION}

\author{
Page 1 of 8
}


Synthesis of dodecylthioether end functionalised poly(methacrylic acid): In a typical preparation, methacrylic acid (MAA, $5 \mathrm{~g}, 58 \mathrm{mmol}$ ), dodecanethiol (DDT, 1.17g, $5.8 \mathrm{mmol}$ ) and 2,2'-azobisisobutyronitrile (AIBN, $0.095 \mathrm{~g}, 0.58 \mathrm{mmol}$ ) were added to EtOH $(25 \mathrm{~mL})$ in a three-necked round-bottomed flask, fitted with a reflux condenser. The temperature of the reaction mixture was maintained at $75{ }^{\circ} \mathrm{C}$ for $5 \mathrm{~h}$ under Nitrogen with magnetic stirring. At the end of this period the reaction mixture was left to cool to room temperature and then the products were isolated by precipitation into cold diethyl ether. The poly(methacrylic acid) (PMAA) was collected by filtration on a Buchner funnel, and the solvent and monomer residues were removed by evaporation to constant mass using a vacuum oven set at $45{ }^{\circ} \mathrm{C}$. Yield $2.3 \mathrm{~g}, 46 \%$. The isolated polymer was soluble in $\mathrm{MeOH}, \mathrm{H}_{2} \mathrm{O}$ and DMSO.

The relatively low yield of this reaction may be attributed to the high level of chain transfer agent introduced. There may be a fraction of low molar mass polymer or oligomer that has been removed during the precipitation step.

\section{Polymer characterization:}

\section{NMR spectroscopy}

${ }^{1} \mathrm{H}$ spectra were recorded on a $400 \mathrm{MHz}$ Bruker DPX-400 spectrometer using $d_{6}$-DMSO as a solvent in a $5 \mathrm{~mm}$ NMR tube. The solvent signal was used as a reference.

\section{Microanalyses}

The C, H, N contents were determined by experiment using a Perkin Elmer 2400 elemental analyser, the $\mathrm{S}$ content was determined by combusting the sample in oxygen and then titration and the $\mathrm{O}$ content was then calculated by difference.

\section{Dual detection size exclusion chromatography}

The instrument package was supplied by Optokem and comprised the following equipment: i) a Jones Chromatography 7600 Series Solvent D-gasser. ii) a Waters 515 HPLC Pump operating at room temperature. iii) a Waters ${ }^{\mathrm{TM}} 717$ plus autosampler with 96 or 48 position sample racks. iv) a column oven set at $40^{\circ} \mathrm{C}$ v) a set of three Styragel HR 2, HR 4 and HR 6 designation $7.8 \times 300 \mathrm{~mm}$ SEC columns. vi) two detectors connected in a serial configuration: a multi-angle light scattering detector (mini-Dawn) supplied by Wyatt Technology and an interferometer refractometer detector (Optilab DSP) supplied by Wyatt Technology.

THF was the mobile phase and the flow rate was $1 \mathrm{~mL} / \mathrm{min}$. (The polymer was first converted to the methyl ester using TMS-diazomethane reagent to render it soluble in THF.) The samples were prepared for injection by dissolving $5 \mathrm{mg}$ of polymer in $1 \mathrm{~mL}$ of HPLC grade THF; $0.2 \mathrm{~mL}$ of this mixture was then injected and data collected for 40 min. 
The polymer was characterised as follows:<smiles>CC(C)(C)CCSC(C)(C)CC(C)(C)C(C)(C)C(=O)O</smiles>

${ }^{1} \mathrm{H}_{\mathrm{NMR}}\left(\boldsymbol{d}_{6}\right.$ DMSO) $\delta$ (ppm): 0.9(b) $\mathrm{CH}_{3}, 1.24\left(\mathrm{CH}_{2}\right)_{9}$ 1.7(b) $\mathrm{CH}_{2}$ (backbone), $2.4 \mathrm{CH}_{2}$ (from DDT), 12.3(b) $\mathrm{CO}_{2} \mathbf{H}$

FT-IR: v(O-H of H-bonded carboxylic acid) $\left(\mathrm{cm}^{-1}\right)$ : 3200 to 2400(b), v(C-H): 2920, $v(\mathrm{C}=\mathrm{O}$ of carboxylic acid): 1695

GPC analysis (methylated polymer): $\boldsymbol{M}_{\mathbf{n}} 5015 \mathrm{~g} / \mathrm{mol} ; \boldsymbol{M}_{\mathbf{w}} 5490 \mathrm{~g} / \mathrm{mol} ; \boldsymbol{M}_{\mathbf{w}} / \boldsymbol{M}_{\mathbf{n}} 1.1$

This corresponds to a degree of polymerization (n) of approximately 50 .

Table S1: \% C, H, S and O content determined from elemental microanalysis

\begin{tabular}{cccccccc}
\hline \multicolumn{2}{c}{$\% \mathrm{C}$} & \multicolumn{2}{c}{$\% \mathrm{H}$} & \multicolumn{2}{c}{$\% \mathrm{O}$} & \multicolumn{2}{c}{$\% \mathrm{~S}$} \\
\hline $\mathrm{T}$ & $\mathrm{F}$ & $\mathrm{T}$ & $\mathrm{F}$ & $\mathrm{T}$ & $\mathrm{F}$ & $\mathrm{T}$ & $\mathrm{F}$ \\
58.7 & 54.4 & 8.0 & 7.7 & 30.1 & 32.0 & 3.0 & 1.3 \\
\hline
\end{tabular}

$\mathrm{T}=$ Theoretical values calculated from the polymer structure $\mathrm{F}=$ Found values determined experimentally for $\mathrm{C}, \mathrm{H}$ and $\mathrm{S}$.

$\mathrm{O}$ content is calculated by difference

Table S2: Recovered polymer composition calculated from ${ }^{1} \mathrm{H}$ NMR and elemental microanalysis data

\begin{tabular}{|c|c|c|}
\hline $\begin{array}{l}\text { Feed ratio } \\
\text { (mole) }\end{array}$ & \multicolumn{2}{|c|}{$\begin{array}{c}\text { Recovered polymer composition (mole) } \\
\text { MAA /DDT calculated from }\end{array}$} \\
\hline MAA/DDT & $\begin{array}{c}{ }^{1} \mathrm{H} \text { NMR } \\
\text { acid polymer }\end{array}$ & $\begin{array}{c}\text { elemental } \\
\text { microanalysis }\end{array}$ \\
\hline $100 / 10$ & $100 / 0 / 7$ & $100 / 0 / 4$ \\
\hline
\end{tabular}

\section{References:}

(1) Cormack, P.A.G, Graham, S., Sherrington, D. C. Macromolecules, 2005, 38, 86-90

(2) Billmeyer, F. W. In Textbook of Polymer Science; Third Edition ed.; John Wiley \& Sons, Inc.: New York, 1984

(3) de la Fuente, J. L., Madruga, E. L. Macromol. Chem. Phys. 2000, 201, 2156-2159 
Synthesis of gold nanoparticles: A known amount (see Table 1) of aqueous polymer solution (dodecylthioether end-functionalized polymethacrylic acid, $M_{\mathrm{w}}=5490 \mathrm{~g} / \mathrm{mol}$, $\left.M_{\mathrm{w}} / M_{\mathrm{n}}=1.1,12 \mathrm{mM}\right)$ was added to an aqueous solution of $\mathrm{HAuCl}_{4}(0.5 \mathrm{mM}, 20 \mathrm{~mL})$ under vigorous stirring. $\mathrm{NaBH}_{4}$ solution $(2 \mathrm{~mL}, 50 \mathrm{mM}$ ) was added one hour after the addition of the polymer. The reducting agent was added rapidly in two aliquots $(1 \mathrm{ml}$ each time). The reaction was allowed to continue overnight under uniform and vigorous stirring.

Transfer of gold nanoparticles into organic solvents: In order to transfer the gold nanoparticles from water to an organic phase, the water-soluble polymer ligand was replaced by an alkanethiol by ligand exchange. Briefly, $20 \mathrm{ml}$ of an ethanolic solution of dodecanethiol (200 $\mathrm{\mu l}$ of dodecanethiol/20 ml of ethanol) was added with stirring to $20 \mathrm{ml}$ of gold nanoparticles in water and vigorous stirring continued overnight. The gold nanoparticles either precipitated at the bottom of the flask or floated at the surface of ethanol/water mixture. The nanoparticles were then transferred to n-hexane and washed three times with distilled water. Traces of water were finally removed by filtering gold nanoparticles in n-hexane over anhydrous sodium sulfate. The gold nanoparticles were dried by solvent evaporation in a rotary evaporator and the excess dodecanethiol was removed by washing the nanoparticles with ethanol (at least three times) without sonication. UV-visible spectra of gold nanoparticles in n-hexane did not differ significantly from those in water, except for some systematic changes due to change in the refractive index of the medium (Figure S1).

TEM: Specimens for inspection by TEM were prepared by evaporating a droplet of the hexane solutions onto a carbon-coated copper mesh grid (Figure 3). Images were obtained using a JEOL-2000 EX transmission electron microscope operating at $220 \mathrm{KV}$.

Calculation of approximate cluster sizes (Table 1): Average particle diameters were determined by TEM and compared with the ideal radii for a given number of atoms as given in Table 2, Ref. 4c. From this we quote the number of Au atoms per cluster in each preparation. This represents an approximation as, even in our unusually uniform preparations, not all clusters contain the same number of atoms. The number of thiol chains was then calculated from AES data - that is, the experimentally determined atomic $\mathrm{Au}: \mathrm{S}$ ratio. 


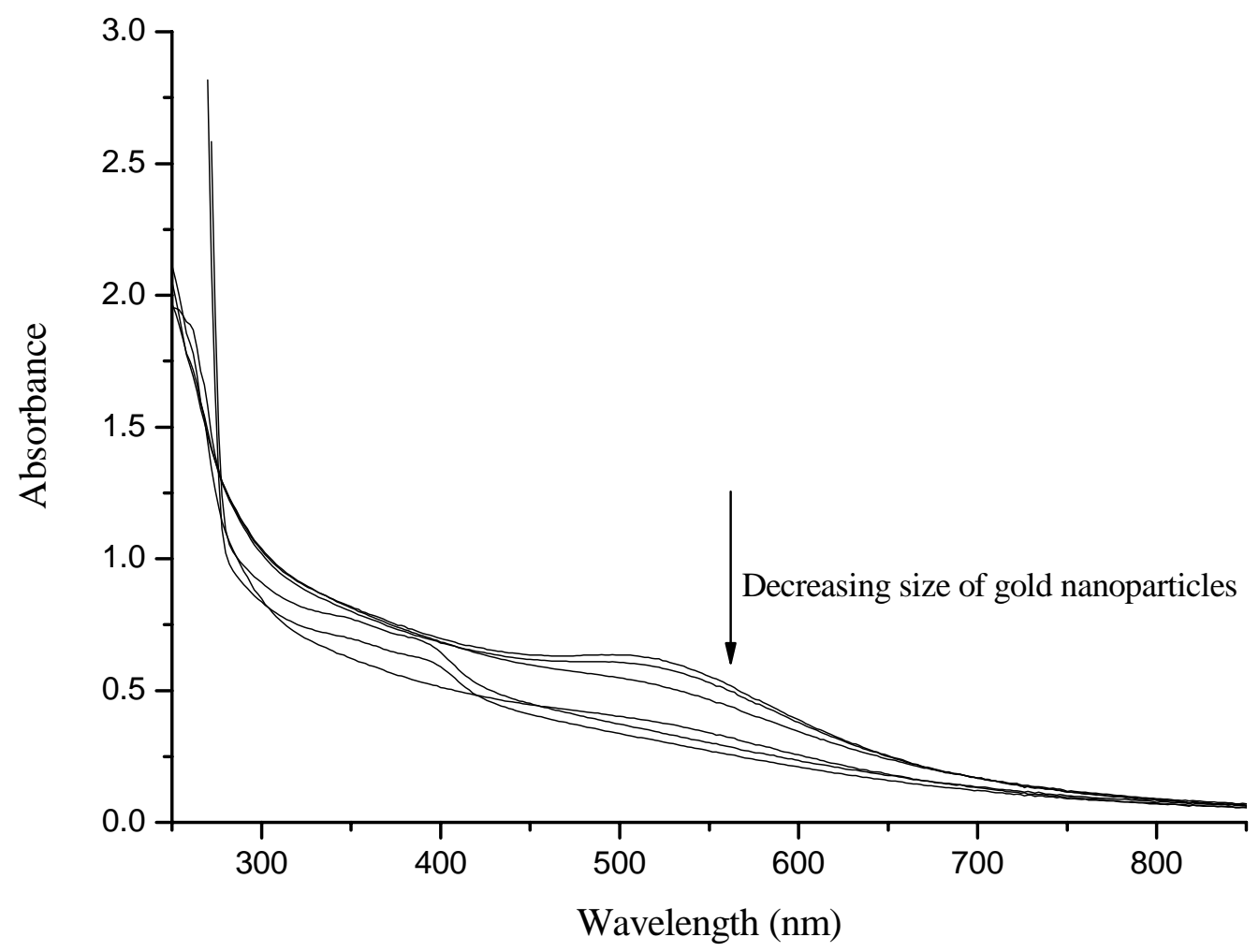

Figure S1. UV-visible absorption spectra of gold nanoparticles transferred into nhexane 

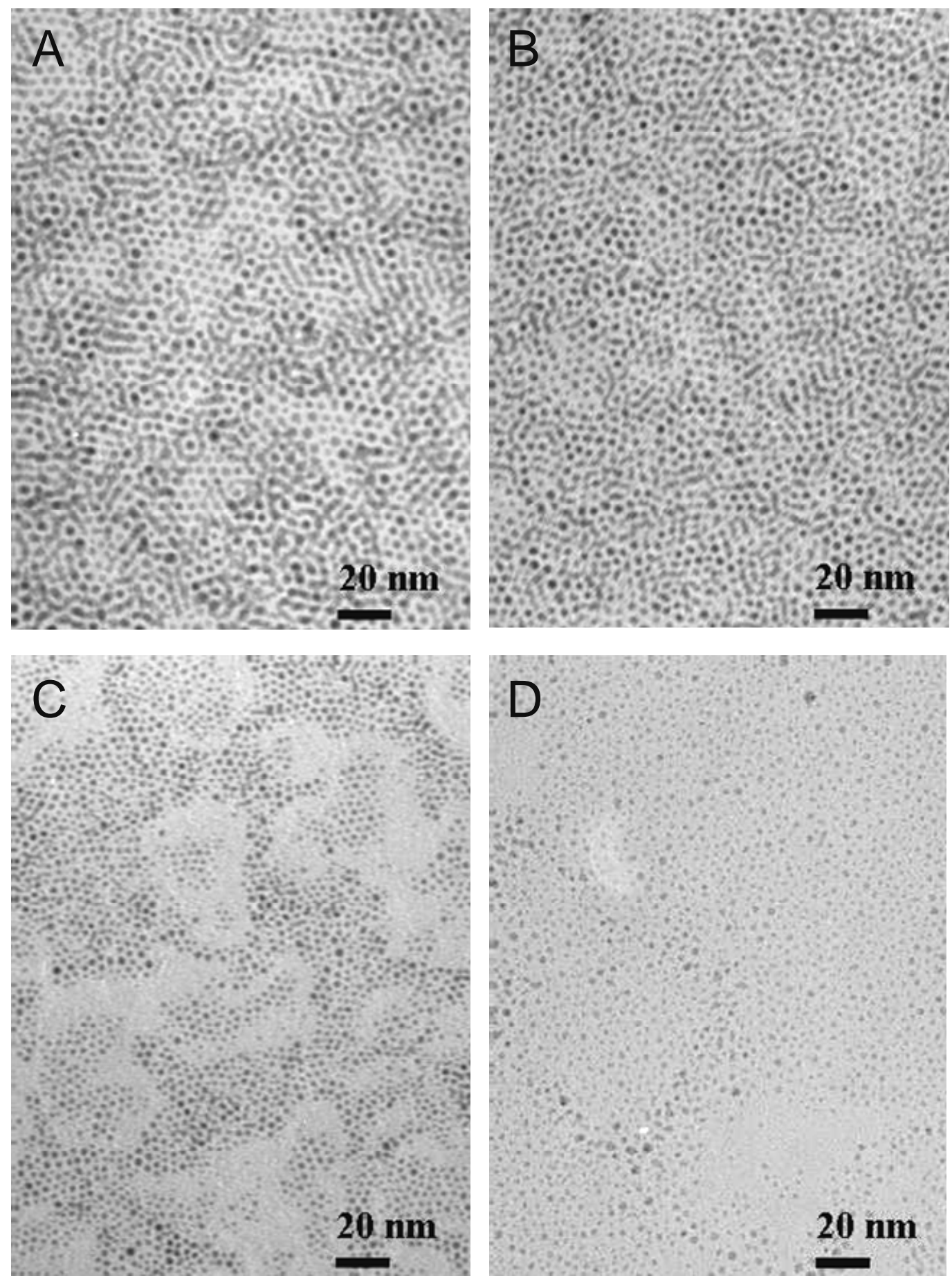

Figure S2. Expanded version of Figure 2 showing particles prepared at four different polymer concentrations (see Table 1 for details). 


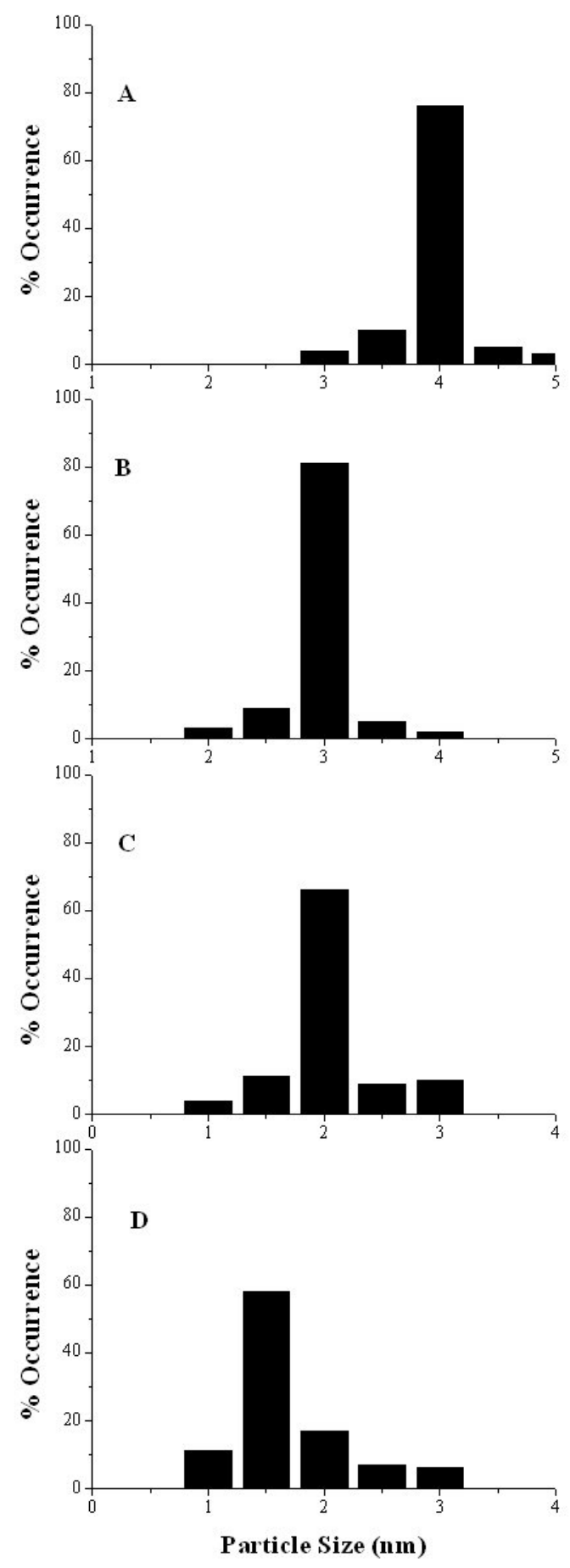

Figure S3. Histogram of particle size distributions for gold nanoparticle samples A-D as calculated from TEM measurements. 


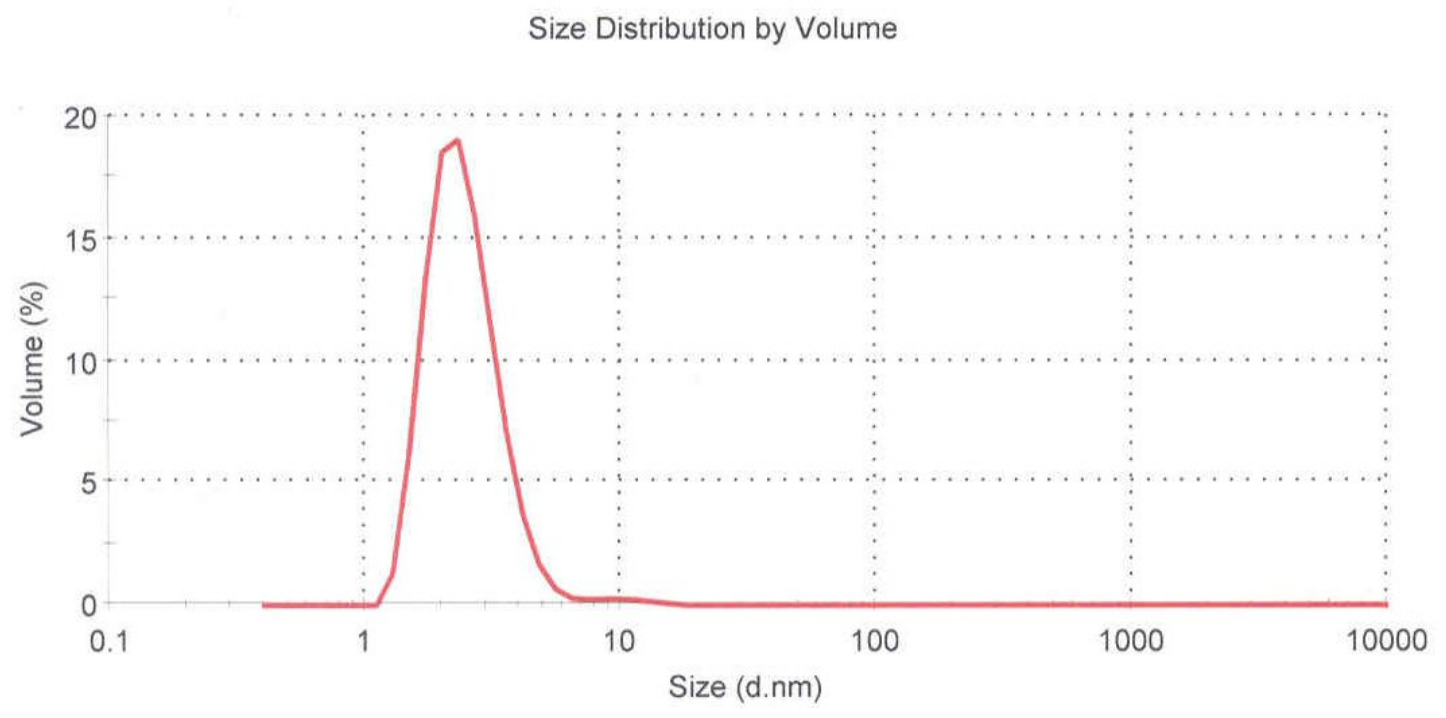

Figure S4. Particle size distribution for poly(methacrylic acid)-stabilized gold nanoparticles as measured using a Malvern Mastersizer 2000 instrument in water at $20{ }^{\circ} \mathrm{C}$. Z-average particle diameter $=2.52 \mathrm{~nm}(98 \%$ vol $)$, peak width $=0.85 \mathrm{~nm}$. Nominal particle diameter from TEM measurements $=2 \mathrm{~nm}$. 